\title{
Malaria Vaccines: Moving Forward After Encouraging First Steps
}

\author{
Tuan M. Tran • Silvia Portugal • Simon J. Draper • \\ Peter D. Crompton
}

Published online: 27 January 2015

(C) Springer International Publishing AG 2015

\begin{abstract}
An effective malaria vaccine that reduces morbidity and mortality and contributes to malaria elimination is a much-needed tool, particularly in endemic areas where health-care delivery and vector control efforts are difficult to sustain. RTS,S/AS01 is likely to be the first licensed malaria vaccine and represents an important step toward malaria control and elimination. However, a partially effective vaccine such as RTS,S/AS01 poses challenges for evaluating the efficacy of second-generation malaria vaccines. Wholesporozoite immunization approaches have shown promising results, inducing sterile immunity in small-scale trials of malaria-naïve adults, but may not achieve durable sterile protection in endemic populations. Vaccines targeting both the preerythrocytic and the erythrocyte-invasive form of the parasite (merozoites) may abrogate breakthrough infections by neutralizing merozoites emerging from infected hepatocytes, whereas vaccines targeting the sexual stages seek to break the transmission cycle. Moving forward, a multi-stage vaccine could be the next step toward malaria elimination and eradication.
\end{abstract}

T. M. Tran $(\bowtie) \cdot$ S. Portugal $\cdot$ P. D. Crompton

Laboratory of Immunogenetics, National Institute of Allergy and

Infectious Diseases, National Institutes of Health, Rockville, MD,

USA

e-mail: tuan.tran@nih.gov

S. Portugal

e-mail: portugals@nih.gov

P. D. Crompton

e-mail: pcrompton@niaid.nih.gov

\section{S. J. Draper}

Jenner Institute, University of Oxford, Old Road Campus Research

Building, Oxford OX3 7DQ, UK

e-mail: simon.draper@ndm.ox.ac.uk
Keywords Plasmodium falciparum $\cdot$ Malaria $\cdot$ Vaccines . Vaccinology $\cdot$ Malaria immunology $\cdot$ Clinical parasitology · Travel medicine $\cdot$ Commentary

Over the last two decades, there has been a marked reduction in malaria morbidity and mortality due to increased global efforts to diagnose and treat malaria cases with artemisininbased combination therapies (ACTs) and to implement effective vector control strategies. From 2000 to 2012, global malaria mortality decreased by $42 \%$ and global incidence rates decreased by $25 \%$, with notable reductions in several subSaharan African countries with high transmission intensity [1]. Yet despite substantial progress toward reducing the overall burden of disease, malaria remains a significant public health threat, afflicting over 200 million people worldwide and causing $\sim 627,000$ deaths in 2012 , primarily in young children suffering from Plasmodium falciparum malaria in sub-Saharan Africa [1]. Moreover, widespread insecticide resistance affecting all major malaria vectors [2], combined with the emergence of artemisinin resistance in P. falciparum [3], threaten to reverse any gains in malaria control achieved thus far. Consequently, the goal of malaria eradication (defined as the sustained reduction to zero of the global incidence of infection by the human malaria parasites) - set forth by Bill and Melinda Gates in 2007 [4] and adopted by the World Health Organization [5] — will be quite challenging with existing control tools and strategies [6], particularly in resource-poor settings with fragile health-care infrastructures. An effective malaria vaccine is widely viewed as a much-needed tool for reducing malaria risk in endemic areas where health-care delivery and vector control strategies are often disrupted by political conflict, natural disasters, and, recently, an ongoing Ebola epidemic which has diverted already scarce resources from malaria control efforts $[7,8]$. However, the complexity of 
the $P$. falciparum life cycle has made vaccine development a daunting task [9]. Moreover, the goal of many malaria vaccines - sterilizing immunity that prevents Plasmodium infection - does not appear to be acquired through natural infection, even after years of repeated P. falciparum exposures [10].

The most clinically advanced malaria vaccine to date is the P. falciparum pre-erythrocytic vaccine RTS,S/AS01, which targets the circumsporozoite protein on the surface of the sporozoite [11]. In a remarkable effort, a phase 3 clinical trial of RTS,S involving 15,460 children across seven African countries showed a vaccine efficacy for clinical malaria of $50 \%$ in older children [11] but only $30 \%$ in infants, the target population [12], without significant protection from severe malaria at 18 months post-vaccination [13] — modest figures relative to highly effective pediatric vaccines such as hepatitis B and measles and well below the anticipated goals for a firstgeneration malaria vaccine set by the Malaria Vaccine Initiative in 2006 [14]. Nonetheless, licensure of RTS,S/AS01 would likely be an important step toward reducing malaria morbidity and may also contribute to malaria elimination efforts in regions of low to moderate $P$. falciparum transmission intensity [13]. The modest protection induced by RTS,S raises interesting questions regarding unknown host immunological or genetic factors that might contribute to the differential efficacy observed among infants [15]. Expanding the scope of biological parameters assayed during both vaccination with RTS,S and the complex host response to Plasmodium infection using comprehensive, unbiased systems approaches may identify correlates of malaria protection and robust immunologic benchmarks to facilitate the development of highly effective second-generation anti-sporozoite malaria vaccines [16]. Another avenue currently being explored as a means of improving the immediate and long-term efficacy of a preerythrocytic subunit vaccine strategy is the combination of RTS,S with an effective T cell-inducing vaccine against the liver-stage form of the parasite [17].

Notably, the licensure of a first-generation vaccine such as RTS,S poses logistical challenges for assessing the efficacy of second-generation vaccines in clinical trials given that a partially effective comparator vaccine would be used in lieu of placebo. A second-generation malaria vaccine candidate that is anticipated to have higher efficacy (i.e., $>70 \%$ efficacy) might require a relatively modest sample size when pitted against RTS,S in a superiority trial; however, any decrease in the absolute difference in efficacy between the two groups would necessitate larger sample sizes to achieve statistical power [18]. Thus, clinical trials for vaccine candidates that are expected to demonstrate only incremental improvements in efficacy relative to an RTS,S comparator would require greater investments of human and financial capital. Among the current malaria vaccine candidates in development, whole-sporozoite immunization approaches such as radiation-attenuated sporozoites (PfSPZ) could potentially advance to phase $2 \mathrm{~b}$ efficacy trials with a presumption of high efficacy based on evidence for sterile protection in previously naïve adults [19]. However, such optimism must be tempered with previous experience with malaria vaccine candidates that performed well in the short term in experimental studies using previously malaria-naïve adults but proved less effective in field studies, possibly due to the genetic diversity of $P$. falciparum parasites in endemic areas [20], immunologic factors specific to malaria-exposed children in endemic areas [15], or the duration of vaccine-induced immunity. Several challenges related to storage and administration of a wholeparasite vaccine also remain to be addressed, and it seems likely that this approach may be best suited in the immediate future to vaccination of travelers or the military, or for use in elimination campaigns in island settings.

Although the above pre-erythrocytic vaccine strategies that target the liver stage aim to induce sterile protection, they are unlikely to achieve complete protection in all individuals, as the escape of just a single sporozoite or liver-stage form can initiate blood-stage infection and therefore clinical malaria. Subunit vaccine strategies that target the erythrocyteinvasive form of the parasite (merozoites), in combination with the pre-erythrocytic forms [21, 22], aim to control and clear the initial blood-stage inoculum emerging from infected hepatocytes [23]. Antibodies elicited by the blood-stage component of the vaccine would neutralize merozoites emerging into the blood from the liver, potentially halting blood-stage replication at a vulnerable choke point prior to the initial round of erythrocyte invasion. Thus, blood-stage vaccines could complement pre-erythrocytic vaccines to achieve the sterile immunity necessary for successful malaria eradication. Short of facilitating sterile immunity, effective blood-stage vaccines may still reduce asexual parasite density, which not only mitigates the clinical manifestations of disease but also could theoretically reduce gametocyte density and thus malaria transmission [24].

Among blood-stage vaccine candidates, the merozoite protein P. falciparum reticulocyte-binding protein homologue 5 (PfRH5) appears to be the most promising given its essential and non-redundant role during erythrocyte invasion, limited genetic diversity, ability to elicit antibodies capable of neutralizing parasite growth, and association with protection from febrile malaria in endemic populations [25-27]. In addition, recent studies characterizing the role of the apical membrane antigen 1/rhoptry neck protein 2 complex [28] and schizont egress antigen-1 [29] contribute to our understanding of blood-stage biology and highlight the potential for a multicomponent approach that targets distinct steps during the erythrocytic cycle. Ideally, future iterations of malaria vaccines adopting a multi-stage approach would also include a transmission-blocking component that prevents the uptake/ development of sexual-stage forms (such as gametocytes or ookinetes) in the mosquito vector [9]. The Pfs 25 ookinete 
surface protein remains the leading candidate antigen for inclusion in such a vaccine, and a number of new formulations are currently entering phase 1 clinical testing [30].

The imminent licensure of the first malaria vaccine represents a major step forward in the fight against malaria. Assessing the efficacy of the next generation of malaria vaccine candidates carries additional challenges, but new vaccine candidates and strategies under development have already demonstrated considerable promise in pre-clinical studies and small clinical trials. A more comprehensive understanding of malaria immunity and the identification of immune correlates of host protection from infection and disease will facilitate the further selection of novel candidate antigens for assessment in a new generation of vaccines. Lastly, iterative testing of multi-component vaccines that target critical preerythrocytic, blood-stage and/or sexual-stage antigens may be required to break the transmission cycle and achieve the sterile protection necessary for malaria eradication.

Acknowledgments Tuan M. Tran, Silvia Portugal, and Peter D. Crompton are supported by the Division of Intramural Research, National Institute of Allergy and Infectious Diseases, National Institutes of Health.

Simon J. Draper is a UK MRC Career Development Fellow, Jenner Investigator, and Lister Institute Research Prize Fellow.

\section{Compliance with Ethics Guidelines}

Conflict of Interest None of the authors have a conflict of interest to declare.

Human and Animal Rights and Informed Consent This article does not contain any studies with human or animal subjects performed by any of the authors.

\section{References}

1. World Health Organization: World Malaria Report 2013: World Health Organization; 2013.

2. World Health Organization: Global Plan for Insecticide Resistance Management in malaria vectors: World Health Organization; 2012.

3. Ashley EA, Dhorda M, Fairhurst RM, et al. Spread of artemisinin resistance in Plasmodium falciparum malaria. N Engl J Med. 2014;371(5):411-23.

4. Gates MF. Malaria Forum Keynote Address. October 17, 2007. http://www.gatesfoundation.org/media-center/speeches/2007/10/ melinda-french-gates-malaria-forum. Accessed October 28, 201.

5. World Health Organization. Global malaria action plan. http://www. rbm.who.int/gmap/. Accessed October 28, 2014.

6. Greenwood B. Can malaria be eliminated? Trans R Soc Trop Med Hyg. 2009;103 Suppl 1:S2-5.

7. Fauci AS. Ebola - underscoring the global disparities in health care resources. N Engl J Med. 2014;371(12):1084-6.

8. Check Hayden E. Ebola obstructs malaria control. Nature. 2014;514(7520):15-6.

9. Riley EM, Stewart VA. Immune mechanisms in malaria: new insights in vaccine development. Nat Med. 2013;19(2):168-78.
10. Tran TM, Li S, Doumbo S, et al. An intensive longitudinal cohort study of Malian children and adults reveals no evidence of acquired immunity to Plasmodium falciparum infection. Clin Infect Dis. 2013;57(1):40-7.

11. Agnandji ST, Lell B, Soulanoudjingar SS, et al. First results of phase 3 trial of RTS,S/AS01 malaria vaccine in African children. N Engl J Med. 2011;365(20):1863-75.

12. SCTP Rts, Agnandji ST, Lell B, et al. A phase 3 trial of RTS,S/AS01 malaria vaccine in African infants. N Engl J Med. 2012;367(24): 2284-95.

13. Rts SCTP. Efficacy and safety of the RTS,S/AS01 malaria vaccine during 18 months after vaccination: a phase 3 randomized, controlled trial in children and young infants at 11 African sites. PLoS Med. 2014;11(7):e1001685.

14. PATH Malaria Vaccine Initiative. Malaria Vaccine Technology Roadmap. August 2006. http://www.malariavaccine.org/files/ Malaria Vaccine_TRM_Final 000.pdf. Accessed October 28, 2014.

15. Daily JP. Malaria vaccine trials- - beyond efficacy end points. N Engl J Med. 2012;367(24):2349-51.

16. Tran TM, Samal B, Kirkness E, Crompton PD. Systems immunology of human malaria. Trends Parasitol. 2012;28(6):248-57.

17. Ewer KJ, O'Hara GA, Duncan CJ, et al. Protective CD8+ T-cell immunity to human malaria induced by chimpanzee adenovirusMVA immunisation. Nat Commun. 2013;4:2836.

18. Fowkes FJ, Simpson JA, Beeson JG. Implications of the licensure of a partially efficacious malaria vaccine on evaluating secondgeneration vaccines. BMC Med. 2013;11:232.

19. Seder RA, Chang LJ, Enama ME, et al. Protection against malaria by intravenous immunization with a nonreplicating sporozoite vaccine. Science. 2013;341(6152):1359-65.

20. Barry AE, Schultz L, Buckee CO, Reeder JC. Contrasting population structures of the genes encoding ten leading vaccine-candidate antigens of the human malaria parasite, Plasmodium falciparum. PLoS One. 2009;4(12):e8497.

21. Heppner Jr DG, Kester KE, Ockenhouse CF, et al. Towards an RTS, S-based, multi-stage, multi-antigen vaccine against falciparum malaria: progress at the Walter Reed Army Institute of Research. Vaccine. 2005;23(17-18):2243-50.

22. Thompson FM, Porter DW, Okitsu SL, et al. Evidence of blood stage efficacy with a virosomal malaria vaccine in a phase IIa clinical trial. PLoS One. 2008;3(1):e1493.

23. Douglas AD, Edwards NJ, Duncan CJ, et al. Comparison of modeling methods to determine liver-to-blood inocula and parasite multiplication rates during controlled human malaria infection. J Infect Dis. $2013 ; 208(2): 340-5$.

24. Sheehy SH, Douglas AD, Draper SJ. Challenges of assessing the clinical efficacy of asexual blood-stage Plasmodium falciparum malaria vaccines. Hum Vaccin Immunother. 2013;9(9):1831-40.

25. Wright GJ, Rayner JC. Plasmodium falciparum erythrocyte invasion: combining function with immune evasion. PLoS Pathog. 2014;10(3): e1003943.

26. Tran TM, Ongoiba A, Coursen J, et al. Naturally acquired antibodies specific for Plasmodium falciparum reticulocyte-binding protein homologue 5 inhibit parasite growth and predict protection from malaria. J Infect Dis. 2014;209(5):789-98.

27. Douglas AD, Williams AR, Knuepfer E, et al. Neutralization of Plasmodium falciparum merozoites by antibodies against PfRH5. J Immunol. 2014;192(1):245-58.

28. Srinivasan P, Ekanem E, Diouf A, et al. Immunization with a functional protein complex required for erythrocyte invasion protects against lethal malaria. Proc Natl Acad Sci U S A. 2014;111(28):10311-6.

29. Raj DK, Nixon CP, Nixon CE, et al. Antibodies to PfSEA-1 block parasite egress from $\mathrm{RBCs}$ and protect against malaria infection. Science. 2014;344(6186):871-7.

30. World Health Organization. Malaria vaccine rainbow tables. [http:/ www.who.int/immunization/research/development/Rainbow tables/ en/]. Accessed November 7, 2014. 